\title{
Characterization of the Timing Homogeneity in a CMOS SPAD Array Designed for Time-Gated Raman Spectroscopy
}

\author{
Jouni Holma, Ilkka Nissinen, Jan Nissinen and Juha Kostamovaara
}

\begin{abstract}
A characterization environment was built to verify the timing characteristics of a single photon avalanche diode (SPAD) array designed for time-gated Raman spectroscopy. The characterization was applied to a $256 \times 16$ SPAD array that employed an on-chip Time-to-Digital converter (TDC) with a 50-100 ps resolution for time-resolving. The timing skew and the time window homogeneity across the array were resolved, moving the time-resolving windows over an optical pulse by picosecond-level delay steps. A typical one 160 ps skew across the array was measured. The TDC time bins had average sizes of 33-144 ps while their deviation across the array was 8-12 ps. The method is applicable to multi-detector time-correlated single photon counting (TCSPC) systems that can finely adjust the delay between the optical pulse and the reference signal.
\end{abstract}

\section{INTRODUCTION}

$\mathbf{T}$ HE recent development of complementary metal oxide semiconductor (CMOS) technology has enabled the monolithic integration of SPADs and peripheral electronics on a single chip. This new level of integration can be utilized in time-correlated single photon counting (TCSPC) measurements, especially when the detector needs multiple channels, such as in fluorescence lifetime imaging [1]. One recent application for a multichannel TCSPC system is timegated Raman spectroscopy [2]-[4].

Raman spectroscopy is based on an inelastic scattering of monochromatic light. In the inelastic scattering of photons, also known as Raman scattering, the wavelength of the scattered photons shifts due to energy transfer between photons and the vibrational, rotational or low-frequency modes of the interacting material. The scattered photons form an intensity distribution depending on the wavelength which is called a Raman spectrum. The shifts of the wavelength depend on the interacting material and thus the Raman spectrum forms a kind of fingerprint of that material [5], [6].

Many Raman spectroscopy applications suffer from a strong fluorescence background that masks the Raman spectrum, as fluorescence can be several orders of magnitude stronger than Raman scattering at the same wavelengths [5]-[7]. Continuous wave $(\mathrm{CW})$ laser illumination, commonly used in Raman spectrometers, accumulates both the Raman spectrum and the fluorescence background, which can fade the Raman spectrum.

However, Raman scattering differs from fluorescence in the time domain, having a lifetime at the scale of picoseconds whereas a lifetime of fluorescence is typically measured from nanoseconds to milliseconds [6], [8]-[10]. The difference in temporal profiles illustrated in Fig. 1 can be distinguished, and this is the basis of a fluorescence suppression method called time-gating. In time-gating, the sample is illuminated by a laser pulse that is narrow in comparison with the lifetime of the fluorescence. When the emission of the sample is recorded only during the Raman emission, the relation between the recorded Raman and fluorescence photons increases [7], [8], [10], [11]. Making the time windows narrower has been shown to significantly improve the performance of the fluorescence suppression [2], [8].

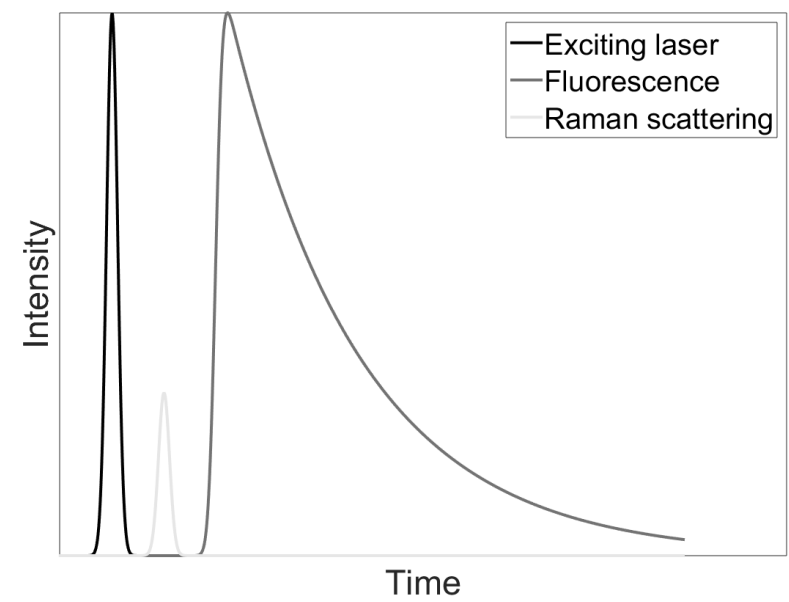

Fig. 1. Illustration of the principle behind time-gated Raman spectroscopy: Raman scattering has a faster response to the exciting laser in comparison with fluorescence emission.

As Raman scattering is a weak phenomenon, a sensitive photon detector is a necessary part of the spectroscope. Charge-coupled device (CCD) arrays are often used as optical sensors in Raman spectroscopes. However, a CMOS SPAD is more suitable for time-gating when it comes to time resolution. Besides this, a SPAD can offer a cheaper, more integrated, smaller-sized and less power-dissipating option for photon expression [3], [6]. A TCSPC setup is a relevant technique for resolving the Raman spectrum, as the technique combines high timing accuracy with single photon detection [12].

The use of SPADs as a detector in time-resolved Raman spectroscopy has been under research for a few years, as the first publication was made in 2011 [11]. Methods of SPAD usage can be divided into two areas: mechanically shifting a single SPAD element across the Raman spectrum, recording each spectrum point separately [2], [13]; and using a SPAD 
array that collects most of the Raman spectrum or the whole spectrum simultaneously [14]-[16]. The first approach has the advantage of having a similar response in different parts of the spectrum when it comes to detector characteristics and time-resolving measurements. The single element has often had an accurate external time-resolving measurement with a resolution below 100 ps or even below 10 ps. The second method measures the spectrum more rapidly, as there are multiple SPAD detectors in line. The array detectors have had partly or fully internal time interval measurements with resolutions at the magnitude of $100 \mathrm{ps}$ or somewhat less at best. A sufficiently wide SPAD array removes the need to shift parts of the system mechanically.

However, the SPAD array faces a few issues that can have an effect on the performance of the Raman spectrometer. As there are many SPADs, each has different noise level [17], called dark count rate (DCR), and slightly varying photon detection probabilities [3]. Besides this, as the SPAD has been shown to emit photons during the avalanche breakdown, optical crosstalk between adjacent SPADs is possible [18]. Finally, some impurities in the SPAD material can hold charge carriers caused by the avalanche and release them when the SPAD is loaded to detect the next photon. Avalanches caused by released charge carriers are called afterpulsing [18].

In an array detector, time-resolving is usually performed by sharing an external electrical reference signal to the elements of the array. The reference signal may reach the elements at different times due to the electrical structure of the array, that forms a systematic timing skew across the array. Besides this, the width of the time gate can vary across the array due to the electrical structure. As the Raman scattering is assumed to reach the SPAD array simultaneously within a narrow time slot, differences in the time-gates across the array and their temporal skew reduce the quality of the Raman spectrum, as shown in [19].

This paper introduces a method for resolving the skew and time window homogeneity of a SPAD array designed for timegated Raman spectroscopy. A characterization environment is built, including a test detector which is a 256x16-SPAD array. The SPAD array employs two on-chip Time-to-Digital Converters (TDC) for time-resolving with a designed resolution of 52 ps. Measurements resolving the timing characteristics of the SPAD array are performed and represented. The paper is organized as follows: a SPAD detector and the test array detector are introduced in II, the built characterization environment is described in III, the measurements are represented in IV and the conclusion is presented in $\mathrm{V}$.

\section{THE TEST SPAD ARRAY}

\section{A. Single photon avalanche diode}

The test detector uses SPADs in photon detection. The operational and design issues concerning these components can be found in [3], [20], for example. To summarize, the photon detection of a SPAD is based on the absorption of a photon to the depletion region of the SPAD that is reverse biased by a voltage above its breakdown voltage. When the reverse bias is above the breakdown voltage, the SPAD is said to be operating in Geiger mode. The photon absorption causes a self-sustaining avalanche breakdown which implies the photon arrival. A neutral area absorption can also be detected when a photo-generated charge carrier is diffused in the depletion region [3]. In order to detect another photon, the SPAD is quenched and loaded into Geiger mode again. Quenching and loading needs external circuitry, such as that represented in Fig. 2. This quenching circuit is easily synchronizable to an external system.

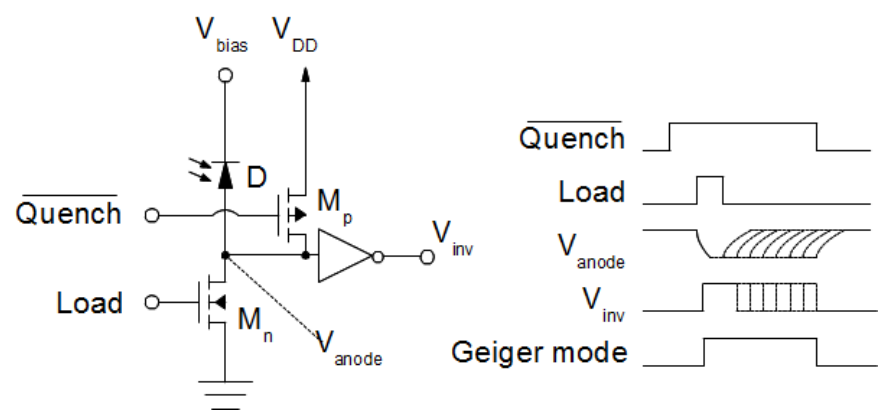

Fig. 2. An active quenching circuitry of a SPAD and timing diagram of the quench signaling. The low-active quench keeps the voltage drop over the SPAD below the breakdown voltage. When the quench is inactive, the load sets the Geiger mode which is held as long as the quench is inactive or a breakdown occurs.

In Fig. 2, the voltages between the $V_{\text {bias }}$ and the ground and between the $V_{\text {bias }}$ and the $V_{D D}$ are above and below the breakdown voltage of the SPAD $D$, respectively. When it is low, the $\overline{\text { quench }}$ opens the pFET $M_{p}$ that keeps the SPAD voltage below the breakdown voltage. When the $M_{p}$ is closed, opening the nFET $M_{n}$ ties the $V_{\text {anode }}$ node into the ground and sets the $D$ to Geiger mode. Closing the $M_{n}$ leaves the $V_{\text {anode }}$ floating and the Geiger mode is held as long as the $M_{p}$ is closed or a breakdown occurs. If the $D$ breaks down, the potential in the floating $V_{\text {anode }}$ rises and quenches the avalanche.

\section{B. SPAD array for time-gated Raman spectroscopy}

The test detector is a 256x16 SPAD array implemented onto an in-house designed integrated circuit (IC) using $350 \mathrm{~nm}$ high voltage AMS CMOS technology. The functional block diagram of the IC is presented in Fig. 3. The IC includes the SPAD array, the quenching circuits of the array, a memory for hot pixel elimination (HPE) configuration, a delay line based time-to-digital converter (TDC) and a register memory for the data generated by the TDC. The external interface of the IC consists of the quench circuit signals, the HPE memory programming interface, the reading interface of the register memory, two start signals for the TDC and a reference clock which stabilizes the TDC resolution.

SPAD elements are $18 \mu \mathrm{m}$ bevelled squares having $35.2 \mu \mathrm{m}$ spectral pitch. Laterally, the elements are organized in pairs having $161 \mu \mathrm{m}$ inter-pair pitch and $32.2 \mu \mathrm{m}$ inside-pair pitch. The overall fill factor of the array is $26 \%$. Each SPAD has a separate quenching circuit, whose implementation is presented in Fig. 2. The load and quench signals represented in Fig. 2 are generated in the pulse generation module of Fig. 3 from four external rising edges. The mutual timings of the rising 


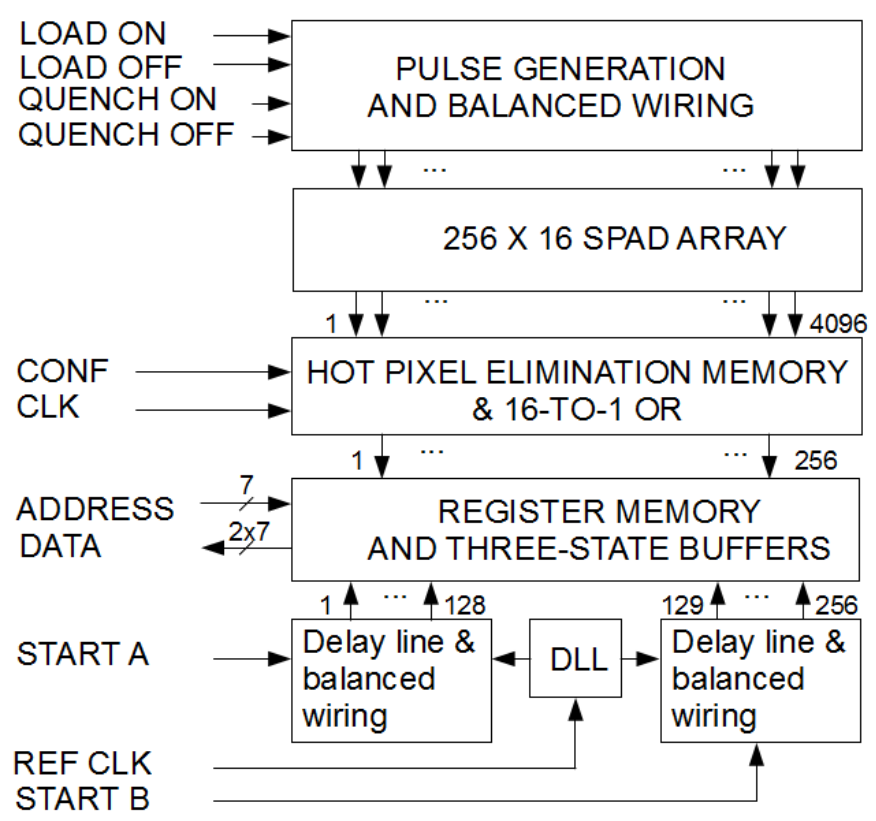

Fig. 3. Block diagram of the 256x16 SPAD array IC designed for time-gated Raman spectroscopy. Each SPAD in the array has a separate quench circuit which receives control signals from the pulse generation module. A row of 16 SPADs has a common output. Two delay lines are used to generate timeresolving codes for SPAD breakdowns. One code per SPAD row is stored in the register memory during a repetition cycle.

edges are presented in Fig. 4. The load and $\overline{\text { quench }}$ signals are tree-wired to the array in order to minimize the systematic timing skew between the different parts of the array.

\section{EXTERNAL}

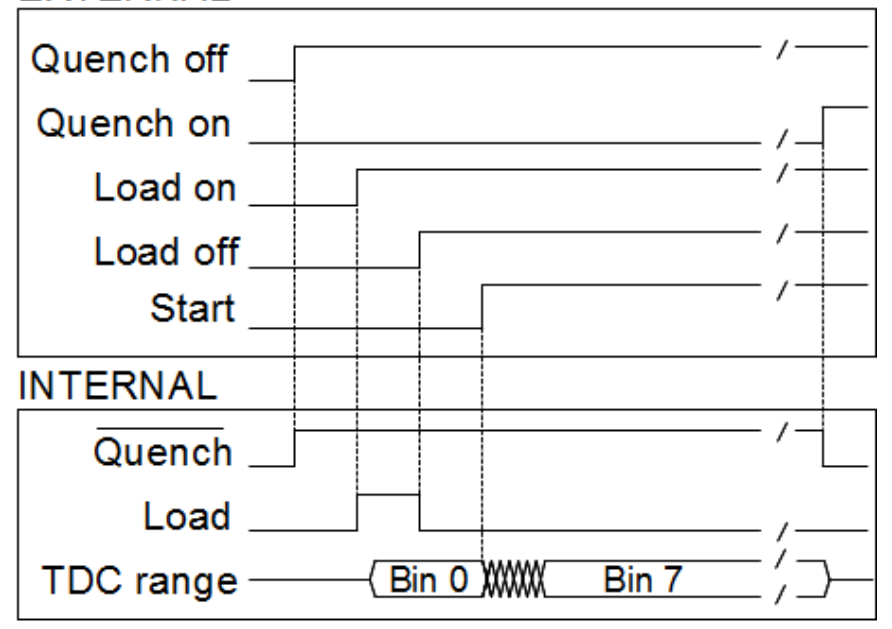

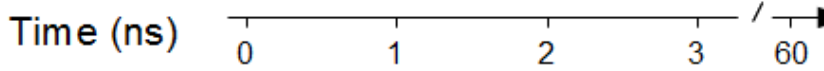

Fig. 4. Timing diagram of the test SPAD array. The quench signaling is generated from four rising edges and the start launches the stopwatch operation of the TDC. The time line is indicative.

The SPAD array has 256 outputs as the outputs of 16 SPAD elements in a row are combined via the OR-port. The OR output rises if any of the input SPADs break down or the $\overline{\text { quench }}$ quenches the Geiger mode. As a wide SPAD array presumably contains a few noisy SPADs, these hot pixels are not wanted to blur the spectrum and can be rejected using the HPE memory: the content of the memory defines which SPAD outputs are led to the OR-ports.

The time-resolving is done by two TDCs that generate time codes for rows 1-128 and 129-256, respectively. The TDC is launched by an external start signal and the TDC code is stored in the register memory when a breakdown occurs. The TDC consists of a delay line of seven delay elements. The start is fed into the delay line and the propagation of the start forms a thermometer code that can be read from the outputs of the delay elements. The thermometer code has eight states - an empty state and seven states for the start propagation phases - and can later be converted into 3-bit binary code. The states are referred to as bins for the remainder of this paper. The timing of the start and the bins in comparison with the quench and the load is illustrated in Fig. 4. The TDC resolution and accurate range consists of six bins in the middle, while the first and the last bins imply that the detection occurs before or after the accurate range, respectively. The thermometer code is tree-wired to the different parts of the array in order to minimize the timing skew.

The TDC compensates thermally caused fluctuation with the delay-locked loop (DLL) represented in Fig. 5. The DLL consists of a replica delay line, a phase detector and a charge pump. The delay of the replica depends on a control voltage that is tuned by the charge pump. An external reference clock leads to the inputs of the phase detector directly and via the replica. The phase difference between the inputs is minimized. The control voltage tuning the replica also tunes the delay lines of the TDC, keeping its performance stable. The resolution of $52 \mathrm{ps}$ is achieved at a $100 \mathrm{MHz}$ reference clock. The function of the TDC is similar to that used in [21] although this TDC has even time bins.

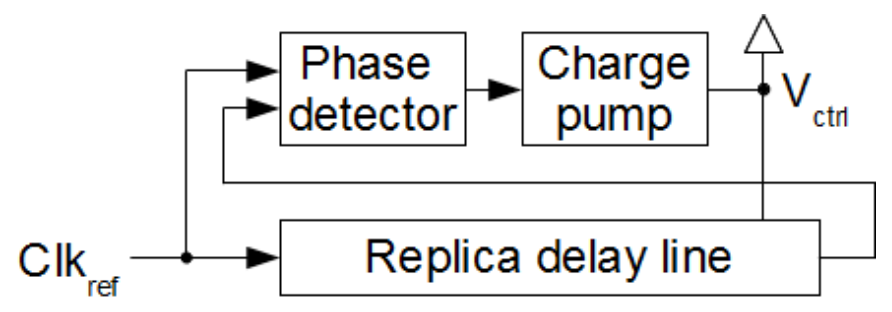

Fig. 5. Block diagram of a delay locked loop.

The thermometer codes are stored in row-specific registers that have tri-stated outputs. The registers can be addressed and read externally. Two adjacent register contents are shown parallel in the data line by one address to speed up the reading process. The address can be changed at a rate of at least 50 MHz.

\section{Characterization ENVIRONMENT}

In order to find the timing skew and the bin homogeneity of the test circuit, a characterization environment was built. Timing skew describes the timing differences of the bins when they reach different parts of the SPAD array. Bin homogeneity depicts the variation of a bin across the array. The skew and bin homogeneity were resolved in a measurement where the 
accurate range of the TDC was moved in the time domain over a narrow laser pulse with small delay steps. In order to resolve the skew, a parametrized model was needed to fit the data. The key parameter of the model is the location at the function of the delay. The skew was resolved by fitting a parametrized Gaussian model for the data, as it included a location parameter and it seemed to fit the data as a moderate approximation. The homogeneity was determined modeling the measurement with a discrete convolution. This section describes the instrumentation and design principles of the characterization environment. The data post-processing and the modeling are detailed in Section IV.

The functional block diagram of the characterization environment is illustrated in Fig. 6. The SPAD array IC is placed on a printed circuit board (PCB) that has power, signal and data connections. The TDC is given a stable external $100 \mathrm{MHz}$ clock for the delay reference. The characterization environment forms a setup where the test circuit is illuminated by a narrow laser pulse to stimulate avalanche breakdowns. A field programmable gate array (FPGA) controls the programming of the HPE memory and the reading of the data from the register memory. The data is transferred to a computer for post-processing. The quenching circuit, the TDC starts and the laser pulse are synchronized to a $100 \mathrm{kHz}$ function generator. The quench signals and the starts are timed according to Fig. 4. The laser pulse is timed to stimulate breakdowns during the accurate range of the TDC. A digital delay generator, programmable delay elements and passive delays - such as coaxial cables and optical fibers - are used in the timing adjustments.

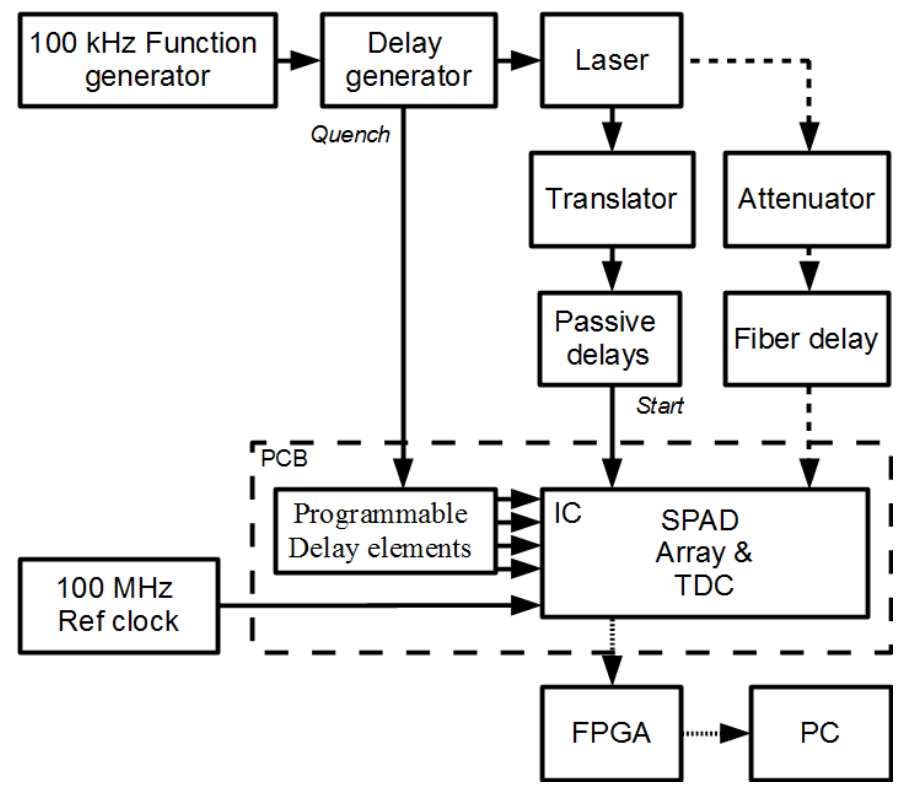

Fig. 6. Functional block diagram of the characterization environment setup. The solid line, dashed line and dotted line arrows represent the signal, the optical and the data progression in the setup, respectively. The IC and programmable delay elements are mounted on a PCB which is delimited by a dashed line.

The laser pulse is attenuated to a level where the detection of more than a photon is unlikely. The TDC start is tied to the laser pulse so that the timing uncertainty between them is minimized because the accurate TDC range is a few hundred picoseconds and a narrow response speeds up the measurements. A gain switched laser [22] was chosen for the illumination as the laser has a low-jitter output trigger. The output trigger is used as a start and translated compatible with the TDC. Coaxial cables and optical fibers define the delay adjustment between the laser and the start. The finest delay tuning is performed with the air-insulated coaxial delay line represented in Fig. 7.

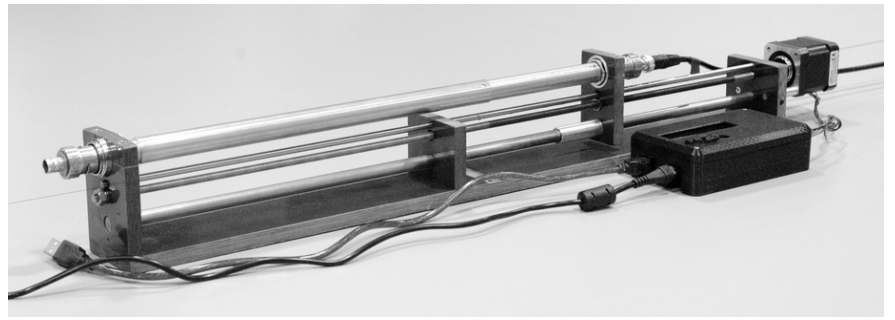

Fig. 7. An air-insulated adjustable coaxial delay line. The length of the delay line is adjusted using a stepper motor that rotates a thread rod. The delay line is used as a low-jitter high-resolution delay.

The characterization environment uses the principles of a TCSPC system: a single photon detector is pulsed by an attenuated laser pulse to enable single photon detection. The detected photons are time-referenced by the TDC bins and a cumulation of the time references forms a histogram [12], [23]. In the characterization environment, the instrumental response function of the system (IRF) is mapped in the histogram. The main components of the IRF include the temporal profile of the laser, the response of the SPAD and the jitter of electronics in different parts of the signal paths. The dispersion of the fibers is also a part of the IRF.

The temporal locations of the photon detection distributions defined by the IRF can vary between the SPAD rows. This variation in the locations is called skew.

In this context, the IRF depicts the temporal precision of the stimulated SPAD breakdowns. The formation of the histogram is illustrated in Fig. 8: photon detections are spread over several TDC bins and cumulation of the measurement forms a histogram. In the characterization environment, the FPGA has a memory where each bin of each SPAD row has a memory location: when a bin from a row is measured, a corresponding memory location is incremented. The data collection is made cumulatively, so that a row-specific memory content forms a histogram after many pulses.

\section{Measurements AND Discussion}

The function of the characterization technique is demonstrated with the built characterization environment in this section. The measurement aims to study the time skew across the SPAD array and the TDC bin homogeneity. In the measurement procedure, the start - and thus TDC range - was moved over the laser pulse in the time domain by small delay steps. A detailed description of the method is presented in Section IV-A. The skew was resolved from the data using Gaussian fitting, presented in Section IV-B. The bin homogeneity was determined modeling the measurement with 


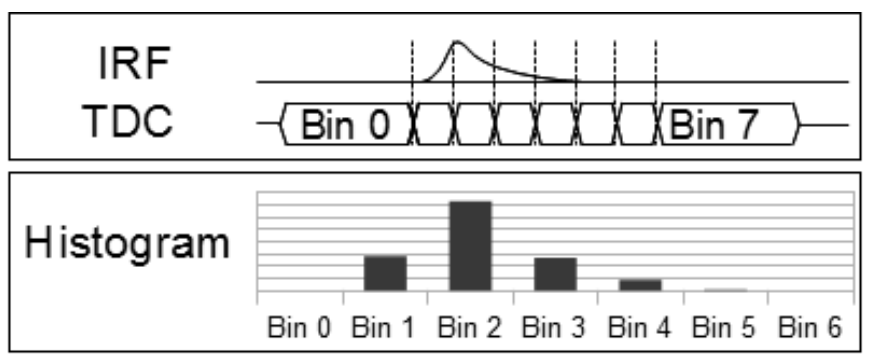

Fig. 8. The detected photons are temporally distributed according to the IRF of the system. When the recording includes a large number of photon detections and the detections are divided according to the TDC bins, a histogram can be formed that is a kind of discrete presentation of the IRF The position of the IRF in comparison with the TDC depends on the timing between the laser pulse and the start. A bin operates like a definite integral.

a discrete convolution in Section IV-C. It will be seen that a convolutional modeling of the measurements offers a simple, efficient and averaging computational tool for resolving the bin widths from the recorded data. The FWHM of the used laser was approximately 100 ps.

\section{A. Measurement setup and moving TDC range}

The laser was attenuated with an optical neutral density filter so that less than $1 \%$ of the repetition cycles caused a stimulated breakdown. The Geiger mode region of the SPADs was defined by the quench signals. Fig. 9 shows a timing sketch of the measurement: the start, and thus the TDC range, was moved over the laser pulse. The laser pulse was a little outside the TDC range at the beginning and end of the measurement. The sliding was performed within the Geiger mode region of the SPADs. Each HPE configuration has a characteristic skew. As an example, all the following measurements have a HPE configuration where is one active SPAD per row.

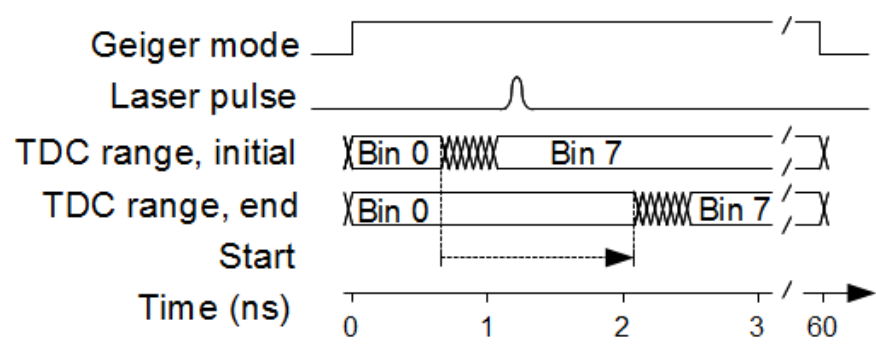

Fig. 9. Timing sketch of the measurement. The start is delayed by small steps in comparison with the laser pulse and the Geiger mode region. As the start is shifted, the TDC range is also shifted. The time scale is indicative.

In the measurement procedure, the Geiger mode region and the laser pulse had fixed delays. The position of the laser pulse defines the position of the photon detection distribution in comparison with the other signals. The start and thus the TDC range was delayed by the coaxial delay line presented in Fig. 7 in $1 \mathrm{~mm}$ steps which corresponds to around a 4 ps signal delay. The laser was pulsed a million times at each delay step at the $100 \mathrm{kHz}$ rate and - based on the TDC bin data - a histogram was built and saved.

Fig. 10 illustrates the bin count development as a function of the delay. Bins 0-6 have very few counts at zero delay, as the stimulated breakdowns occur after the TDC range at the beginning of the measurement, as shown in Fig. 9. The small offset at bin 0 is due to the dark counts which occur between the beginning of the Geiger mode and the start. The size of the bin and the number of dark counts occurring within the bin have an approximately linear relationship when only single dark counts are recorded. Thus, the offset in bin 0 slightly increases as bin 0 extends with the delay, as seen in Fig. 9. When the whole delay range is concerned, the IRF of the system is mapped in each bin. A single delay step shows how the detections are distributed between the bins. When the delay is increased, the maximum of the photon detections moves from bin 6 to bin 0 . The height of the curve depends on the size of the bin.

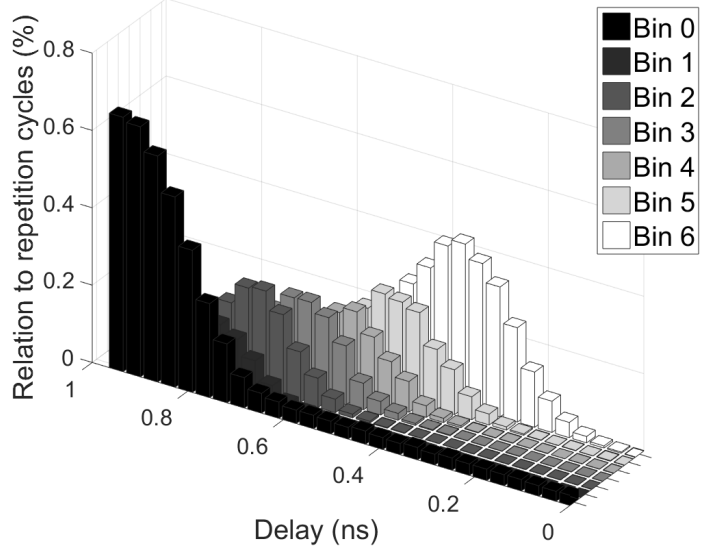

Fig. 10. TDC histograms of the function of delay in a SPAD when the start is delayed by small steps in comparison with the Geiger mode and the laser pulse. For illustration purposes, this figure shows every tenth delay step from the sliding TDC range measurement.

\section{B. Gaussian fitting and skew}

The skew definition was performed using Gaussian curve fitting. The fitting included statistical noise removal and Gaussian pulse fitting to bins 1-6. The Gaussian model was chosen for the fitting because the IRF was assumed to have dominant Gaussian components. The Gaussian model included three parameters: amplitude, mean and width.

The skew was resolved by collecting the mean parameters of the Gaussian models across the SPAD array and forming a curve within a bin. Fig. 11 shows a skew across the SPAD array within bin 1. As the IC has two separate TDCs, there are two skew graphs covering the first and the second halves of the array. The skews from bins 1-6 are collected in Table I in Section IV-D.

\section{Convolutional model and window homogeneity}

The measurement procedure described in Section IV-A is modeled as a discrete convolution, as the delaying of the start is performed in steps. Fig. 8 illustrates the forming of a histogram based on the overlap between the IRF and a bin when the number of detections is high. A bar of the histogram 


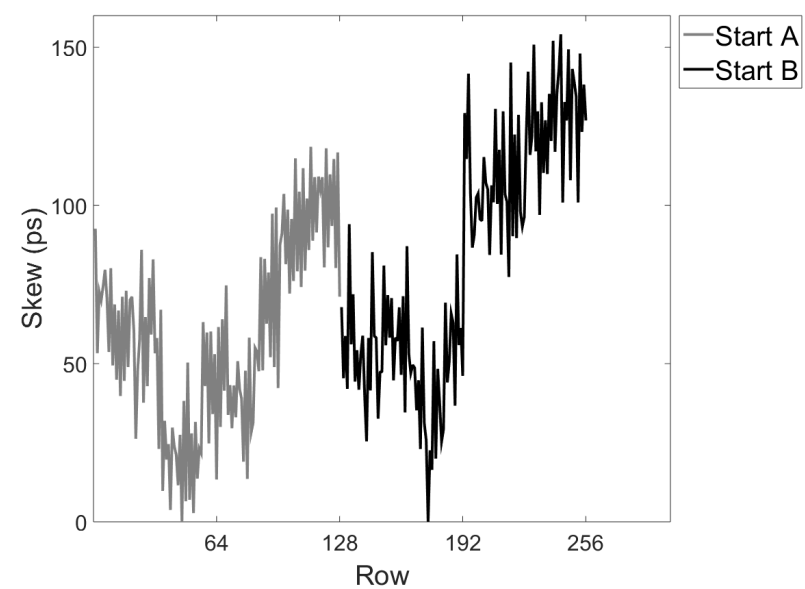

Fig. 11. The skew of the SPAD array in the TDC bin 1 based on the mean parameters of the Gaussian models. The array has two TDCs which each cover half of the array.

can be described as a definite integral whose boundaries are defined by the size and position of the bin. As the start is delayed, the TDC range is shifted in comparison with the IRF, as illustrated in Fig. 10. In the discrete convolution, the function of the definite integral can be performed with a unit height rectangle impulse response. Thus, in terms of the convolution, the bin is modeled as a unit height rectangle impulse response and the IRF acts as a stimulus. The width $m$ of the impulse response is a multiple of the delay step.

The bin widths can be resolved from the measurement results, as the discrete convolution is a product of the stimulus and the impulse response. A unit height rectangle impulse response can be utilized in the calculation of the impulse response width as described in the following paragraphs.

Equation 1 describes a discrete convolution $y[j]$ with a finite length stimulus $x[k]$ and a finite length unit rectangle pulse impulse response $h[k]$ whose lengths are $n$ and $m$, respectively.

$$
\begin{aligned}
y[j] & =\sum_{k=-\infty}^{\infty} x[k] h[j-k], \\
x[k] & = \begin{cases}>0, & 0 \leq k<n-1 \\
0, & \text { elsewhere }\end{cases} \\
h[k] & = \begin{cases}1, & 0 \leq k<m-1 \\
0, & \text { elsewhere }\end{cases}
\end{aligned}
$$

The discrete convolution $y[j]$ has a length $m+n-1$. The sum of the convolution $y[j]$ is expressed in Equation 2.

$$
\begin{aligned}
\sum_{j=0}^{m+n-2} y[j] & =\underbrace{x[0]}_{\mathrm{y}[0]}+\underbrace{x[0]+x[1]}_{\mathrm{y}[1]}+\ldots+\underbrace{x[n-1]}_{\mathrm{y}[\mathrm{m}+\mathrm{n}-2]} \\
& =m *(x[0]+\ldots+x[n-1]) \\
& =m \sum_{k=0}^{n-1} x[k]
\end{aligned}
$$

Thus, the width $m$ of the impulse response can be resolved, as shown in Equation 3.

$$
m=\frac{\sum_{j=0}^{m+n-2} y[j]}{\sum_{k=0}^{n-1} x[k]}
$$

Although the IRF of the characterization environment is not known completely, the bin widths can be resolved when Equation 3 is applied. The sum of the convolution describes the number of hits in a bin during the whole delay range. The sum of the stimulus describes the average number of stimulated breakdowns in each delay step.

The convolutional model was applied to the measurement procedure. Fig. 12 illustrates the bin sizes and positionings across the SPAD array. The skew can also be seen. Bins 0 and 7 are cut in Fig. 12 as their widths are not reasonable in comparison with bins 1-6 and are irrelevant from the application point of view. A numeral analysis of the time window homogeneity is represented in Table I in Section IV-D.

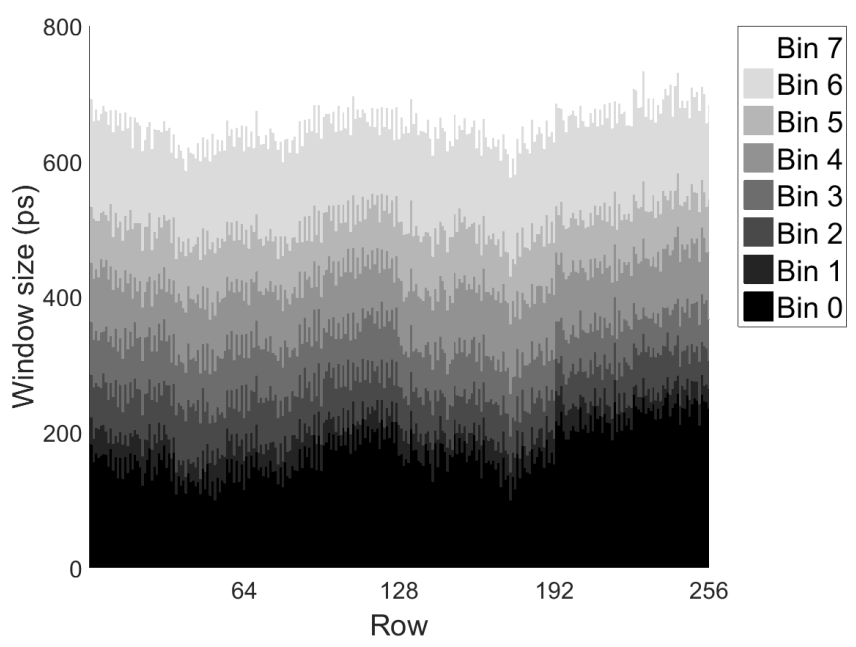

Fig. 12. Bins are stacked in the same time scale. The bin homogeneity and the skew can be illustrated simultaneously when a discrete convolution model is applied to the data: the bin data is integrated over the delay range, divided by the average hit count per delay step and multiplied by the width of the delay step. Bins 0 and 7 are cut due to their superior widths in comparison with bins 1-6.

\section{Summary of the results and discussion}

The timing skew of the SPAD array and the time bin homogeneity were resolved by delaying the start signal by small steps and collecting a histogram at each step, detailed in Section IV-A. The skew was measured by fitting a Gaussian curve into the data and comparing the mean values of the Gaussian model across the array, detailed in Section IV-B. The skew is represented bin-wise in Table I. An alternative skew measure was given from a discrete convolution model where the sizes and positions of the bins were resolved, as derived in Section IV-C. The convolutional model represents the skew as the centers of the bins. The bin widths were solved through the convolutional model. The skew is typically 160 
ps in the Gaussian model and a little less with the discrete convolution model. The differences between these models are described below. The bin widths have around 10 ps variation.

TABLE I

A TYPICAL BIN-WISE REPRESENTATION OF THE SKEW AND THE BIN HOMOGENEITY ACROSS THE SPAD ARRAY. THE SKEW IS DERIVED FROM THE SAME DATA BY GAUSSIAN CURVE FITTING AND CONVOLUTIONAL MODELING. THE SKEW DESCRIBES THE VARIATION OF THE BIN CENTER ACROSS THE SPAD ARRAY.

\begin{tabular}{ccccc}
\hline \hline & \multicolumn{2}{c}{ Skew (ps) } & \multicolumn{2}{c}{ Window size (ps) } \\
& Gaussian fitting & Convolutional model & $\mu$ & $\sigma$ \\
\hline Bin 1 & 154 & 151 & 33 & 8.6 \\
Bin 2 & 160 & 147 & 61 & 10.4 \\
Bin 3 & 151 & 145 & 69 & 10.7 \\
Bin 4 & 155 & 145 & 87 & 9.2 \\
Bin 5 & 171 & 148 & 80 & 8.7 \\
Bin 6 & 179 & 158 & 144 & 10.1 \\
\hline \hline
\end{tabular}

The essence of the characterization environment is a sliding of the time resolving range over the optical stimulus. The process is not dependent on the instrumentation insofar as the system has a TCSPC capability and the delay between the optical pulse and the reference can be finely adjusted over a certain range. Changing the functional components of the system varies the IRF that influences the measurement time. However, the shape of the IRF is secondary.

Intuitively, the quality of the Gaussian parameters is higher, when the fit error is smaller. When the IRF can be approximated by a Gaussian model and the bin width is narrow in comparison with the FWHM of the IRF, the data is more suitable for Gaussian fitting. Increasing the bin width changes the shape of the data away from the Gaussian form. On the other hand, a wide IRF causes inaccuracy, as the deviation of the model parameters increases with the IRF. The Gaussian model can be applied even if the sliding of the TDC range over the optical pulse is not complete.

The discrete convolution between a stimulus and a unit height impulse response is a model of the measurement procedure. This model has two averaging components: the pulse count within a delay step and the size of the delay step. Increasing the number of pulses decreases the deviation between the photon detection histograms within the measurement. The size of the delay step can be reduced, which increases the number of delay steps. The shape of the stimulus has statistical variation, which influences the quality of the process. Due to the analogy between the discrete convolution and the measurement process, the timing performance of a multi-detector TCSPC can be easily visualized.

When these two data processing methods are compared, the convolution method presumably gives more accurate timing homogeneity information and demands less computational resources in comparison with the Gaussian model. However, the Gaussian model can be formed with less data points in comparison with the convolution method.

\section{CONClusion}

A characterization environment resolving the timing characteristics of a time-resolving SPAD array designed for timegated Raman spectroscopy has been built. For time-resolving, the SPAD array had an internal TDC that had six accurate time windows. The characterization environment was designed to perform TCSPC functionality with the SPAD array as a detector. The timing characteristics were studied by changing the delays between the external timing signals. The timing skew and the time window homogeneity was resolved after data post-processing. The minimum measured timing skew across the array was $151 \mathrm{ps}$. The sizes of the time windows had average values from $33-144$ ps. The deviation inside the time windows across the array was $8-12$ ps. The timing characterization method is applicable to other multi-detector TCSPC systems that have a fine delay adjustment in the electrical signal path. Our future plans are to find ways to improve the Raman spectrum quality. The most important issue is to arrange the optical stimulus within the TDC range despite the skew. We are also looking for methods for correcting the distortion caused by the time window inhomogeneity and the skew.

\section{ACKNOWLEDGMENT}

This work has been funded by the Academy of Finland, Center of Excellence in Laser Scanning Research under Contract 272196, Contract 255359 and Contract 283075. The authors would like to thank the Academy for the financial support.

\section{REFERENCES}

[1] R. M. Field and S. Realov and K. L. Shepard A $100 \mathrm{fps}$, Time-Correlated Single-Photon-Counting-Based Fluorescence-Lifetime Imager in $130 \mathrm{~nm}$ CMOS, IEEE Journal of Solid-State Circuits, 49(4), pp. 867-880, 2014.

[2] J. Kostamovaara and J. Tenhunen and M. Kögler and I. Nissinen and J. Nissinen and P. Keränen, Fluorescence suppression in Raman spectroscopy using a time-gated CMOS SPAD, Optics Express, 21(25), pp. 31632-31645, 2013.

[3] D. P. Palubiak and M. J. Deen, CMOS SPADs: Design Issues and Research Challenges for Detectors, Circuits, and Arrays, IEEE Journal of Selected Topics in Quantum Electronics, 20(6), pp. 409-426, 2014.

[4] D. Bronzi and F. Villa and S. Tisa and A. Tosi and F. Zappa, SPAD Figures of Merit for Photon-Counting, Photon-Timing, and Imaging Applications: A Review, IEEE Sensors Journal, 16(1), pp 3-12, 2016.

[5] E. B. Hanlon and R. Manoharan and T- W. Koo and K. E. Shafer and J. T. Motz and M. Fitzmaurice and J. R. Kramer and I. Itzkan and R. R. Dasari and M. S. Feld, Prospects for in vivo Raman spectroscopy, Physics in medicine and biology, 45(2), pp. 1-59, 2000.

[6] D. Wei and S. Chen and Q. Liu, Review of Fluorescence Suppression Techniques in Raman Spectroscopy, Applied Spectroscopy Reviews, 50(5), pp. 387-406, 2015.

[7] P. Matousek and M. Towrie and C. Ma and W. M. Kwok and D. Phillips and W. T. Toner and A. W. Parker, Fluorescence suppression in resonance Raman spectroscopy using a high-performance picosecond Kerr gate, Journal of Raman Spectroscopy, 32(12), pp. 983-988, 2001.

[8] D. V. Martyshkin and R. C. Ahuja and A. Kudriavtsev and S. B. Mirov, Effective suppression of fluorescence light in Raman measurements using ultrafast time gated charge coupled device camera, Review of scientific instruments, 75(3), pp. 630-635, 2004.

[9] A. Laubereau and D. von der Linde and W. Kaiser, Direct Measurement of the Vibrational Lifetimes of Molectiles in Liquids, Physical review letters, 28(18), pp. 1162-1165, 1972.

[10] R. P. Van Duyne and D. L. Jeanmaire and D. F. Shriver, ModeLocked Laser Raman Spectroscopy-A New Technique for the Rejection of Interfering Background Luminescence Signals, Analytical Chemistry, 46(2), pp. 213-222, 1974.

[11] I. Nissinen and J. Nissinen and Antti-Kalle Länsman and L. Hallman and J. Kostamovaara and J. Tenhunen and M. Kögler, A Sub-ns Time-gated CMOS Single Photon Avalanche Diode Detector for Raman Spectroscopy, Proceedings of the ESSDERC, pp. 375-378, 2011. 
[12] W. Becker, Advanced Time-Correlated Single Photon Counting Techniques, Springer, Berlin, Germany, 2005.

[13] Z. Li and M. J. Deen, Towards a portable Raman spectrometer using a concave grating and a time-gated CMOS SPAD, Optics Express, 22(15), pp. 18736-18747, 2014.

[14] I. Nissinen and J. Nissinen and J. Holma and J. Kostamovaara, A TDCbased 4x128 CMOS SPAD Array for Time-Gated Raman Spectroscopy, IEEE European Solid-State Circuits Conference, pp. 139-142, 2014.

[15] Y. Maruyama and J. Blacksberg and E. Charbon, A 1024x8 700ps time-gated SPAD line sensor for laser raman spectroscopy and LIBS in space and rover-based planetary exploration, IEEE Journal of Solid-State Circuits, 49(1), pp. 179-189, 2013.

[16] N. Krstajic and R. Walker and J. Levitt and S.P. Poland and D. Li and S. Ameer-Beg and R.K. Henderson, A 256 x 8 SPAD line sensor for time resolved fluorescence and raman sensing, ESSCIRC, pp. 143-146, 2014.

[17] F. Zappa and S. Tisa and S. Cova and P. Maccagnani and D. B. Calia and R. Saletti and R. Roncella and G. Bonanno and M. Belluso, Singlephoton avalanche diode arrays for fast transients and adaptive optics, IEEE Transactions on Instrumentation and Measurement, 55(1), pp. 365374, 2006.

[18] F. Zappa and S. Tisa and A. Tosi and S. Cova, Principles and features of single-photon avalanche diode arrays, Sensors and Actuators A: Physical, 140(1), pp. 103-112, 2007

[19] I. Nissinen and J. Nissinen and P. Keränen and A. Länsman and J. Holma and J. Kostamovaara, A 2 x (4) x 128 Multitime-Gated SPAD Line Detector for Pulsed Raman Spectroscopy, IEEE Sensors Journal, 15(3), pp. 1358-1365, 2015.

[20] S. Cova and M. Ghioni and A. Lacaita and C. Samori and F. Zappa, Avalanche photodiodes and quenching circuits for single-photon detection, Applied Optics, 35(12), pp. 1956-1976, 1996

[21] I. Nissinen and Antti-Kalle Länsman and J. Nissinen and J. Holma and J. Kostamovaara, A TDC-based 4x128 CMOS SPAD Array for TimeGated Raman Spectroscopy, Proceedings of the ESSCIRC, pp. 291-294, 2013

[22] L. Hallman and J. Huikari and J. Kostamovaara, A high-speed/power laser transmitter for single photon imaging applications, Sensors, IEEE, pp. 1157-1160, 2014.

[23] H. Wang and Y. Yang and Z. Huang and H. Gui, Instrument for RealTime Measurement of Low Turbidity by Using Time-Correlated Single Photon Counting Technique, IEEE Transactions on Instrumentation and Measurement, 64(4), pp. 1075-1083, 2015.

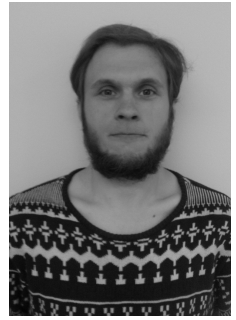

Jouni Holma was born in Liminka, Finland, in 1986. He received an M.Sc. in Electrical Engineering from the University of Oulu in 2013 and has been a Research Scientist in the Circuits and Systems Research Unit at the University of Oulu since then. His research interests include embedded testing and characterization environment development for time-gated SPAD arrays designed for Raman spectroscopy.

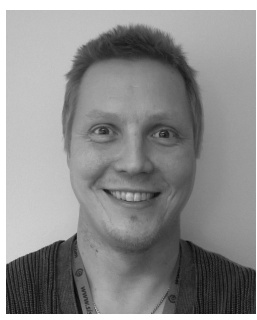

Ilkka Nissinen (M09) was born in Oulu, Finland, in 1976. He received M.Sc. Eng., Licentiate of Tech. and Dr. Tech. degrees in electrical engineering from the University of Oulu, Finland, in 2002, 2007 and 2011 , respectively. He has been a Research Scientist in 2002 - 2011 and Postdoctoral Researcher since 2011 in the Circuits and Systems Research Unit at the University of Oulu. His research interests include the design of time interval measurement architectures for the integrated receiver of pulsed time-of-flight laser rangefinders and the development of time-gated single photon avalanche diode arrays for Raman spectroscopy. $\mathrm{He}$ has designed or co-designed approximately 10 ASICs during his career and has authored or co-authored 24 scientific papers.

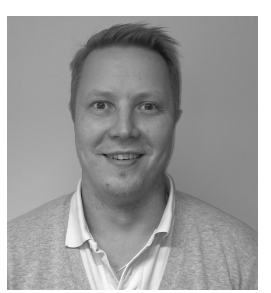

Jan Nissinen was born in Oulu, Finland, in 1976. He received M.Sc. Eng., Licentiate of Tech. and Dr.Tech. degrees in electrical engineering from the University of Oulu, Finland, in 2002, 2007 and 2011, respectively. He has been a Research Scientist in 2002-2011 and Postdoctoral Researcher since 2011 in the Circuits and Systems Research Unit in University of Oulu. His research interests include the design of analog and mixed-signal integrated circuits for pulsed time-of-flight laser rangefinders and for pulsed Raman spectroscopy.

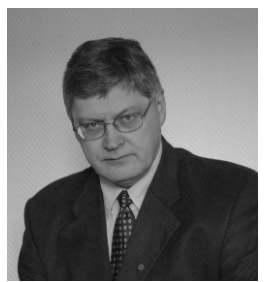

Juha Kostamovaara (M85, S13) holds a full professorship in electronics at the University of Oulu, Finland. In 2007 he was nominated to the Academy professorship position by the Academy of Finland, a position he holds till 2017. His main interests are in the development of pulsed time-of-flight devices and circuits and their applications in electronic and optoelectronic measurements. 